\title{
PULSIVE FEEDBACK CONTROL FOR STABILIZING UNSTABLE PERIODIC ORBITS IN A NONLINEAR OSCILLATOR WITH A NON-SYMMETRIC POTENTIAL
}

\author{
G. $\operatorname{LITAK}^{1}$, M. ALI ${ }^{2}$, L.M. SAHA ${ }^{3}$ \\ ${ }^{1}$ Department of Applied Mechanics, Technical University of Lublin, \\ Nadbystrzycka 36, PL-20-618 Lublin, Poland \\ ${ }^{2}$ Department of Mathematics, Faculty of Mathematical Science, \\ University of Delhi, Delhi 110007, India \\ ${ }^{3}$ Zakhir Husain College, University of Delhi, Delhi 110002, India
}

\begin{abstract}
We examine a strange chaotic attractor and its unstable periodic orbits in case of one degree of freedom nonlinear oscillator with non symmetric potential. We propose an efficient method of chaos control stabilizing these orbits by a pulsive feedback technique. Discrete set of pulses enable us to transfer the system from one periodic state to another.
\end{abstract}

Keywords: nonlinear vibration, chaos control 
A chaotic motion appearing in specific physical systems may show various positive and negative effects [Thomsen 2003] depending on applications. Opportunity of its control triggered a new filed of nonlinear research [Fradkov \& Evans 2005]. Starting with a strange chaotic attractor to system control is a comfort situation because of infinite number of unstable periodic orbits included in it. This novel idea of using unstable periodic orbits to chaos control has been invented by Ott, Geborgi and Yorke [1990] (OGY method) and applied to many physical systems [Fradkov \& Evans 2005]. Namely, Ott et al. [1990] have shown that one can convert a chaotic attractor to any of a large number of possible attracting time-periodic motions by making only small time-dependent perturbations of available system parameters. In other words one can stabilize any unstable periodic orbit included in a strange chaotic attractor. The next important step was the selfcontrolling feedback method introduced by Pyragas [1992], where the small perturbations were continuous in time.

Impulsive methods for dynamical systems' control and synchronization are some known approaches in the field of chaos [Yang et al. 1997, Osipov et al. 1998a, Osipov et al. 1998b, Sun \& Zhang 2004, Sun et al. 2004, Khadra et al. 2005]. It was used successfully for controlling Rössler system [Yang et al. 1997] and the Duffing oscillator [Osipov et al. 1998a] to periodic motions. More recent paper about impulsive control was more successful in establishing more conservative and sufficient conditions for the stabilization and synchronization of Lorenz systems via impulsive control. In their recent work, Sun \& Zhang [2004], presented some new theorems on the stability of impulsive control systems, which was applied successfully to the Chua's oscillator. Based on stability theory of impulsive differential equation and new comparison theory, the authors of [Sun et al. 2004] studied the chaos impulsive synchronization of two coupled chaotic systems using the unidirectional linear error feedback scheme. Moreover, in the most recent work [Khadra et al. 2005], this approach was used with non-linear partial differential equations. The authors determined a criterion for the solutions of these partial differential equations to be equi-attractive in the large and estimated the basin of attraction in terms of the impulse durations and the magnitude of the impulses. In our paper, we apply the same impulsive method with a linear feedback strategy based on the knowledge of unstable periodic orbit embedded within the chaotic attractor of the original system.

We start from a single degree of freedom system subjected to an external excitation with a non-symmetric stiffness given by the following equation:

$$
\ddot{x}+\alpha \dot{x}+\delta x+\gamma x^{2}=\mu \cos \omega t
$$

where $x$ is a displacement, $\alpha \dot{x}$ is linear damping, $\mu \cos \omega t$ is an external excitation, while $\delta x$ and $\gamma x^{2}$ are linear and quadratic force terms.

The above equation has been extensively studied by Thompson \& Hunt [1989], who found chaotic behavior there and examined transitions to chaos through a a global homoclinic bifurcation and a cascade of period doubling bifurcations just before escape from the potential well. Such systems (Eq. 2) have been also a subject of studies for many other researchers, inspired by possible applications in description of mostly mechanical systems [Szabelski \& Samodulski 1985, Szemplinska-Stupnicka \& Rudowski 1993, SzemplinskaStupnicka 1995, Rega et al. 1995, Rand 2003, Litak et al. 2005, Litak et al. 2006] and the catastrophe theory [Poston 1978]. They were also linked to possible meta-stable states of atoms and they appeared in problems within the elastic theory [Thompson 1989, Thompson \& Hunt 1984]. 
The above equation is equivalent to the following autonomous system of three first-order differential equations:

$$
\begin{aligned}
& \dot{x}=y \\
& \dot{y}=-\alpha y-\delta x-\gamma x^{2}+\mu \cos (\omega z) \\
& \dot{z}=1,
\end{aligned}
$$

where $z=t$, therefore, whenever one attempts to integrate the system (Eq. 2), one must pay attention to the fact that the initial conditions must be such that $z_{0}=t_{0}$. From the recent paper by Litak et al. [2006] it is clear that the system given by Eq. 1] exhibits a chaotic motion and a strange attractor for the parameters' values $\alpha=1.0, \delta=1.0$, $\omega=0.85, \gamma=1.089$ and $\mu=0.608$. For practical reasons of system control in relatively large values of variables we have rescaled the variables by acquiring the following variables' changes:

$$
\begin{aligned}
& x \rightarrow \frac{x}{100}, \\
& y \rightarrow \frac{y}{100} .
\end{aligned}
$$

After the above transformation the system (Eq. 2) remains the same while the parameters' values of $\gamma$ and $\mu$ would become 0.01089 and 60.8 , respectively. The strange attractor of a chaotic motion of this system has been shown in Fig. 1. We can start examining the system Eq. 2 looking for an unstable periodic orbit:

$$
X^{*}(t)=\left[x^{*}(t), y^{*}(t), z^{*}(t)\right]
$$

embedded within its chaotic attractor of period $2 \pi / \omega$. This orbit has been obtained numerically by a method of recurrence and has been shown in Fig. 2. The basic idea of recurrence is to wait two successive iterations of the designed Poincare map of sections to fall in a sufficiently small neighborhood. In our case, for the sake of more accuracy, we have used the same concept but with a little modification. Given the dimension of the phase space and the range of the variables, we where able to determine a rectangle in the phase space where points of the unstable periodic orbit are suspected to be within it. Using Mathematica, we were able to develop a code that can detect a smaller rectangle within the previous one in which points of the unstable periodic orbit are lying within it. This was done by dividing the previous rectangle over a net of 10000 smaller and identical rectangles then integrating the given flow starting with each mesh on the net and finding the mesh at which the smallest recurrence occurs. Repeating the same procedure successively finitely many times, we were able to determine a point (mesh) at which an arbitrarily small recurrence occurs. Integrating and plotting the orbit initialized at this mesh over the same period of the Poincare map, would give us the best approximation of the required unstable periodic orbit of the given period.

Using a feedback technique, we have been able to stabilize the unstable period-1 orbit of this system which was embedded within its chaotic attractor (Fig. 2). This has been done by adding a small perturbation:

$$
\epsilon\left(X(t)-X^{*}(t)\right)
$$


to the considered system (Eq. 21). The stabilized period-1 orbit of the given system is shown in Fig. 3. Starting with the same initial conditions as in unperturbed system (Fig. 11) we have easily obtained periodic motion of the system. In this case control parameter was assumed to be $\epsilon=-0.5$. For smaller $|\epsilon|$ the same final aim have been obtained but in relatively longer time. In fact the resulting behavior of the system does not change qualitatively while changing a value of $\epsilon$.

An alternative method is to apply the same feedback technique to the given system but on a discrete scale as pulses (pulsive feedback technique). For instance, one does not need to keep watching and observe the dynamics of the system all the time in order to supply the system with necessary perturbations continuously, instead this task is done at predetermined discrete and equal time intervals proportional to the period of the unstable periodic orbit that we wish to stabilize. In the example given below, each pulse is set to last for a time equal to the pre-assigned integration step and several pulses are provided to the system per period. This would eliminate all waste of unnecessary energy required in the original continuous feedback technique and make it an optimal technique as the cost of control is minimized. For the integration, we used a Runge-Kutte method of second order with a step size equal to $2 \pi /(1000 \omega)$ i.e. one cycle of period $\tau=2 \pi / \omega$ was divided into 1000 equal time intervals for the integration purpose.

Applying the pulsive feedback technique to the examined system (Eqs 2-3) in case of the time interval between two successive pulses was $\pi /(3 \omega)$ produced interesting results.

Beside the fact that we have been able to stabilize the same unstable period- 1 orbit of this system, we have also been able to transfer the system from one periodic state to another by varying the pulses strength $\epsilon$. Figures 4a-c show stabilized period-1 $(2 \pi / \omega)$, period-2 and period-4 orbits of the system (Eq. 2) using the pulsive feedback technique with $\epsilon=-0.05, \epsilon=-0.03$ and $\epsilon=-0.009$, respectively. In fact, we have found that the system (Eq. 2) with pulsive feedback undergoes a period doubling bifurcation as the pulses strength $\epsilon$ increases in the interval $[-0.05,0]$. A bifurcation diagram of our system with pulsive feedback control is shown in Fig. 迎 with $\epsilon \in[-0.05,0]$. This is the principal result of our present investigations. We would like to stress that it is very promising for engineering practice and real life experiments as it enables switching between one state and another of the same system by simple controlling the pulses' strength provided for the system.

Here using the same predetermined unstable periodic orbit of period embedded within the chaotic attractor, we have not only been able to stabilize this orbit but also a variety of other unstable periodic orbits, as shown in the bifurcation diagram (Fig4d). The period and nature of the stabilized orbit depend entirely on the perturbation's coefficient (which is supposed to be small). Different stabilized orbits are shown in Figs 4a-c In order to stabilize an unstable periodic orbit of a dynamical system, knowledge of the same orbit is not necessary, instead, one may make use of any other unstable periodic orbit of lower period available of the same system.

The above result may be also considered as an advantage of the pulsive feedback technique over the well known feedback technique [Pyragas 1992, Fradkov \& Evans 2005] which does not enable such switching from one state a dynamical system to another without re-engineering the whole method in order to suit a particular state. In this paper we have applied this technique to a system with single non-symmetric well [Thompson \& Hunt 1989] potential. However we claim that it should work successfully for any system with a period doubling bifurcation cascade. Note also, our approach to system control 
differs from so called impulsive control methods [Yang et al. 1997, Osipov et al. 1998a, Osipov et al. 1998b] where the main issue was suppressing chaotic motion. In their cases the system is stabilized by adequately strong impulsive signal which drive it to periodic motion of various properties. These periodic orbits, in contrast to the present consideration, were not related to unstable periodic orbits of a strange attractor. In our case the pulsive control method is based on unstable periodic orbit and it is making use of a feedback control in discrete way and the stabilized orbits are strongly related to other unstable orbits of a strange attractor with multiple period.

\section{Acknowledgments}

GL would like to acknowledge a partial support from the Polish State Committee of Scientific Research. 


\section{References}

Fradkov, A.L. \& Evans, R.L. [2005] "Control of chaos: Methods and applications in engineering", Annual Reviews in Control 29, 33-56.

Khadra, A. Liu X. \& Shen, X. [2005] "Impulsive control and synchronization of spatiotemporal chaos", Chaos, Solitons $\&$ Fractals, 26, 615-636.

Litak, G., Syta, A., Borowiec, M. \& Szabelski, K. [2005] "Transition to chaos in the self-excited system with a cubic double well potential and parametric forcing", Int. J. Non-Linear Mech., submitted.

Litak G, Syta A \& Borowiec M. [2006] "Suppression of chaos by weak resonant excitations in a nonlinear oscillator with a non-symmetric potential", Chaos, Solitons $\&$ Fractals, in press.

Osipov, G., Glatz, L. \& Troger, H. [1998a] "Suppressing chaos in the Duffing oscillator by impulsive actions", Chaos Solitons \& Fractals 9, 307-321.

Osipov, G.V., Kozlov, A.K. \& Shalfeev V.D. [1998b] "Impulsive control of chaos in continuous systems", Phys. Lett. A 247, 119-128.

Ott, E., Geborgi, C. \& Yorke, J.A. [1990] "Controlling chaos", Phys. Rev. Lett. 64 1196-1199.

Poston, T. \& Stewart J. [1978] Catastrophe theory and its applications, (Pitman, London)

Pyragas, K. [1992] "Continuous control of chaos by self-controlling feedback", Phys. Lett. A $170,421-428$.

Rand, R.H. [2003] Lecture notes on nonlinear vibrations (Ithaca:The Internet-First University Press, Ithaca), http://www.tam.cornell.edu/randdocs/.

Rega, G., Salvatori, A. \& Benedettini, F. [1995] "Numerical and geometrical analysis of bifurcation and chaos for an asymmetric elastic nonlinear oscillator", Nonlinear Mechanics 7, 249-272.

Sun, J.T, Zhang, Y.P. \& Wu, Q.D. [2002] "Impulsive Control for the Stabilization and Synchronization of Lorenz Systems". Phys. Lett. A, 298, 153-160.

Sun, J.T \& Zhang, Y.P. [2004] "Impulsive Control and Synchronization of Chua's Oscillators". Mathematics and Computers in Simulation, 66 499-508.

Sun, J.T., Zhang, Y.P., Qiao F. \& Wu, Q.D. [2004] "Some Impulsive Synchronization Criterions for Coupled Chaotic Systems via Unidirectional Linear error Feedback Approach", Chaos, Solitons \& Fractals, 19, 1049-1055.

Szabelski, K. \& Samodulski, W. [1989] "Drgania ukladu z niesymetryczna charakterystyka sztywnosci przy parametrycznym i zewnetrznym wymuszeniu", Mechanika Teoretyczna $i$ Stosowana $23,223-238$.

Szemplińska-Stupnicka, W. \& Rudowski, J. [1993] "Bifurcattions phenomena in a nonlinear oscillator: approximate analytical studies versus computer simulation results", Physica D 66, 368-380.

Szemplińska-Stupnicka W. [1995] "The analytical predictive criteria for chaos and escape in nonlinear oscillators: A survey", Nonlinear Dynamics 7 129-147.

Thompsen, J.J. [2003] Vibrations and stability (Springer, Berlin).

Thompson, J.M.T. \& Hunt, G.W. [1984] Elastic instability phenomena, (Wiley, Chichester). 
Thompson, J.M.T. [1989] "Chaotic Phenomena Triggering the Escape from a Potential Well", Proceedings of the Royal Society of London A 421, 195-225.

Yang, T., Yang, C-M. \& Yang, L-B. [1997] "Control of Rössler system to periodic motions using impulsive control methods", Phys. Lett. A 232, 356-361. 


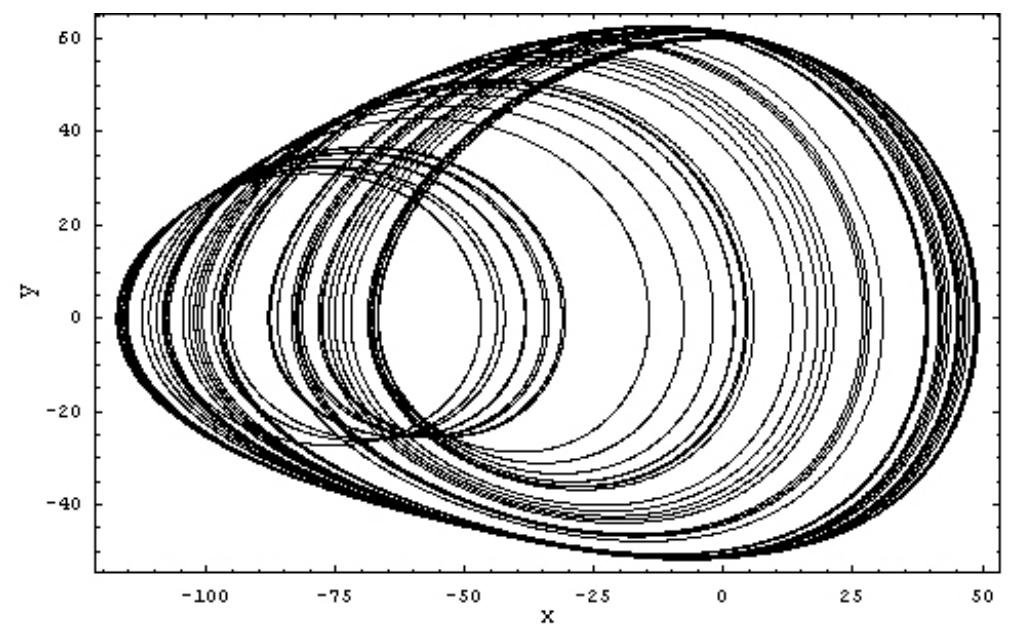

Figure 1: The chaotic strange attractor of the system (Eq. 2) for the parameters values $\alpha=1.0, \delta=1.0, \omega=0.85, \gamma=0.01089$ and $\mu=60.8$.

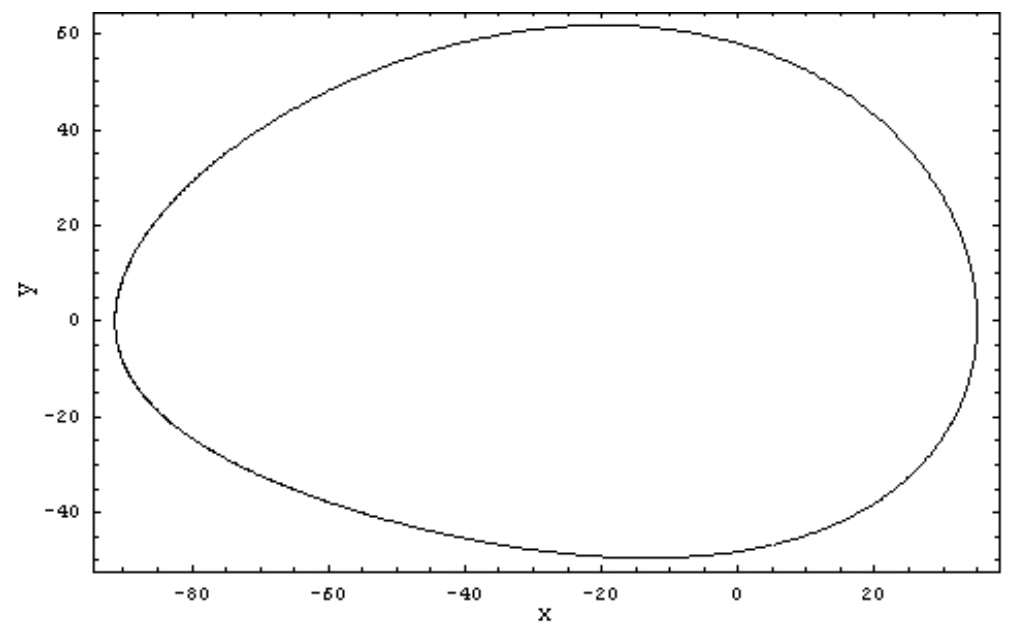

Figure 2: An unstable period-1 orbit of the strange attractor Fig. 1. 


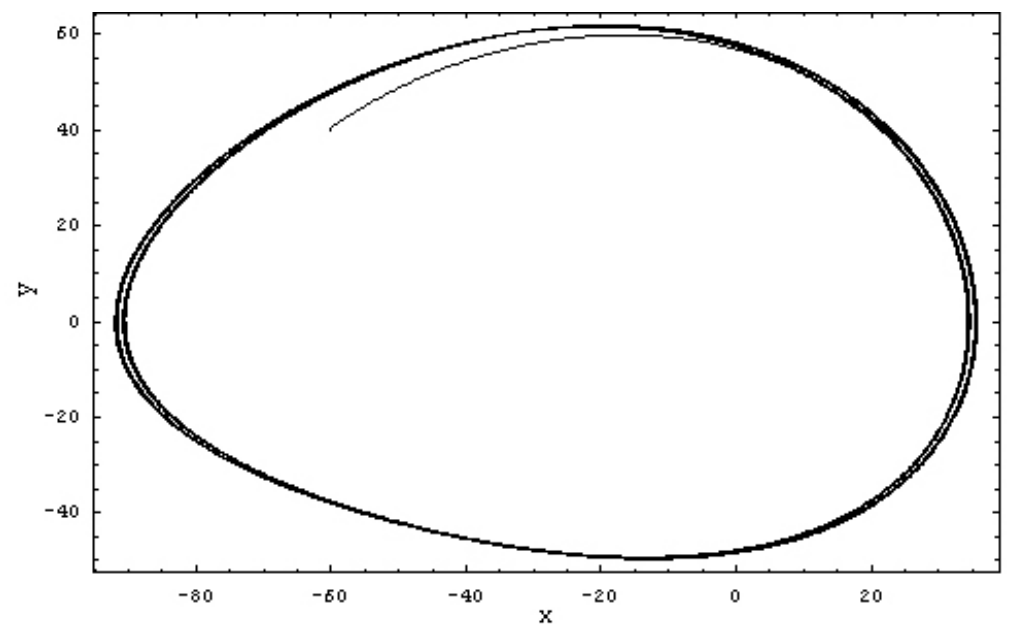

Figure 3: Stabilized period-1 orbit of system (Eqs. 2) using the feedback technique with $\epsilon=-0.5$. 
(a)

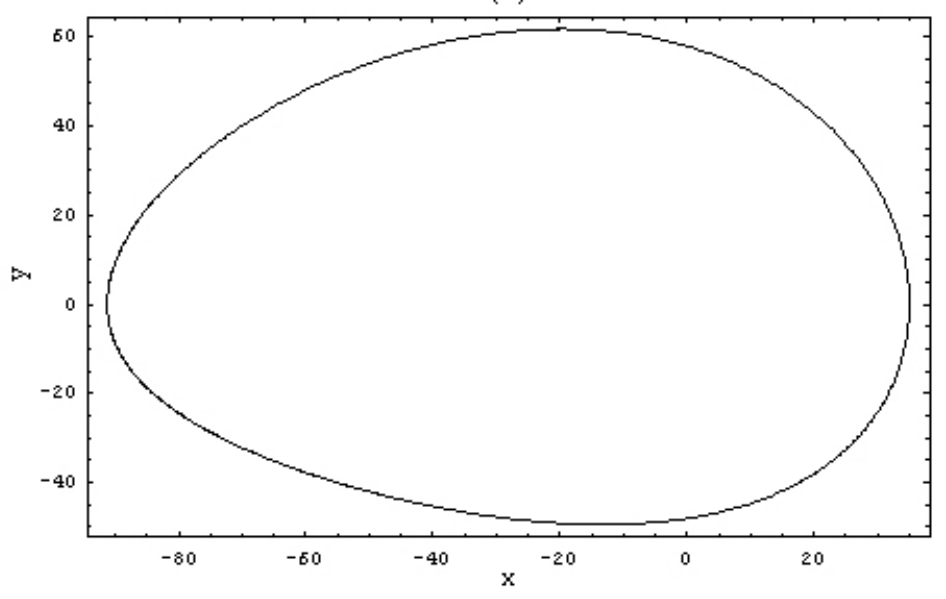

(b)

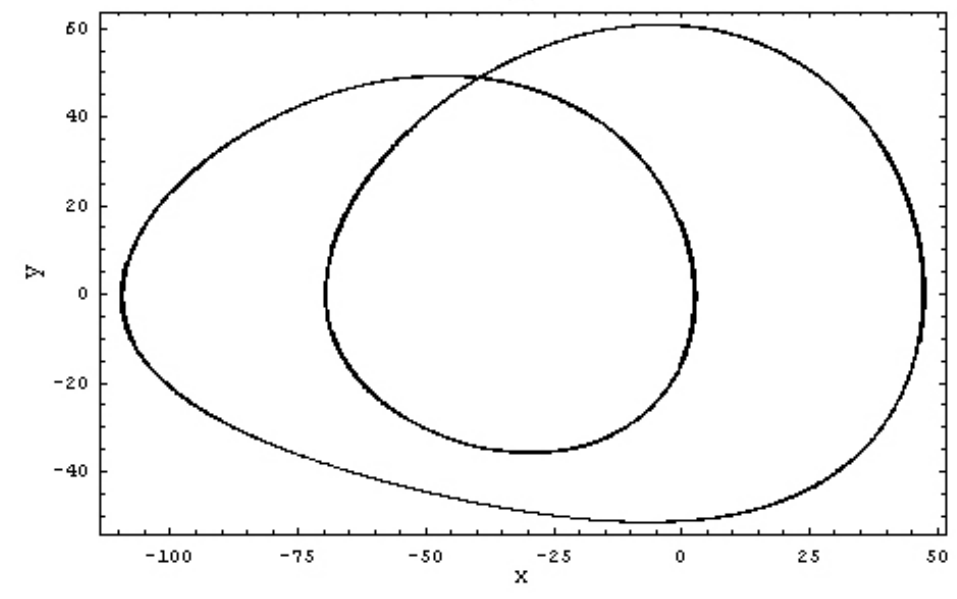

Figure 4: Stabilized period-1 (Fig 因), period-2 (Fig 因) and period-4 (Fig 因) orbits of system (Eq. 2) using pulsive feedback technique with $\epsilon=-0.05,-0.03$ and -0.009 , respectively. A bifurcation diagram with pulsive feedback versus $\epsilon \in[-0.05,1]$. 
(c)

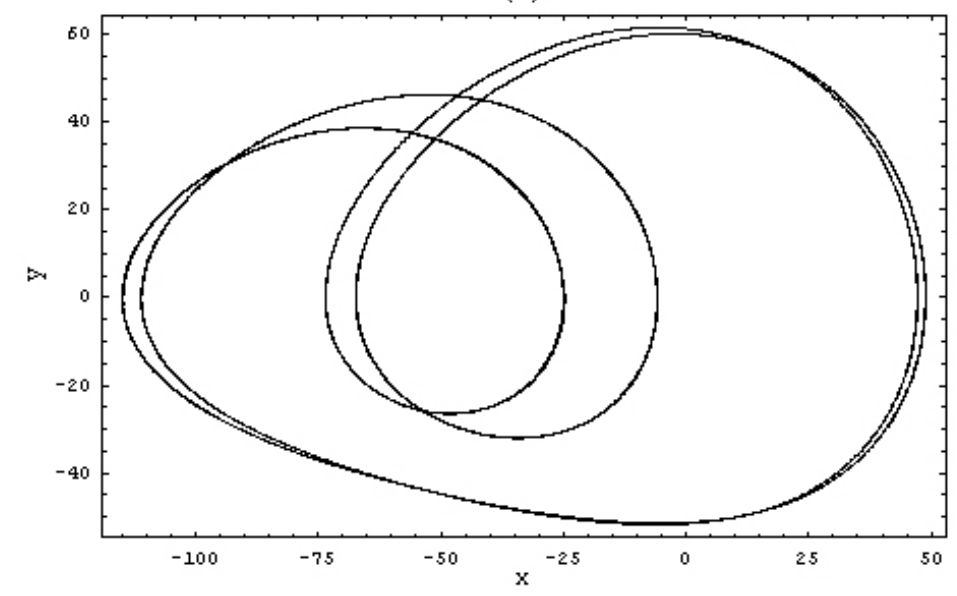

(d)

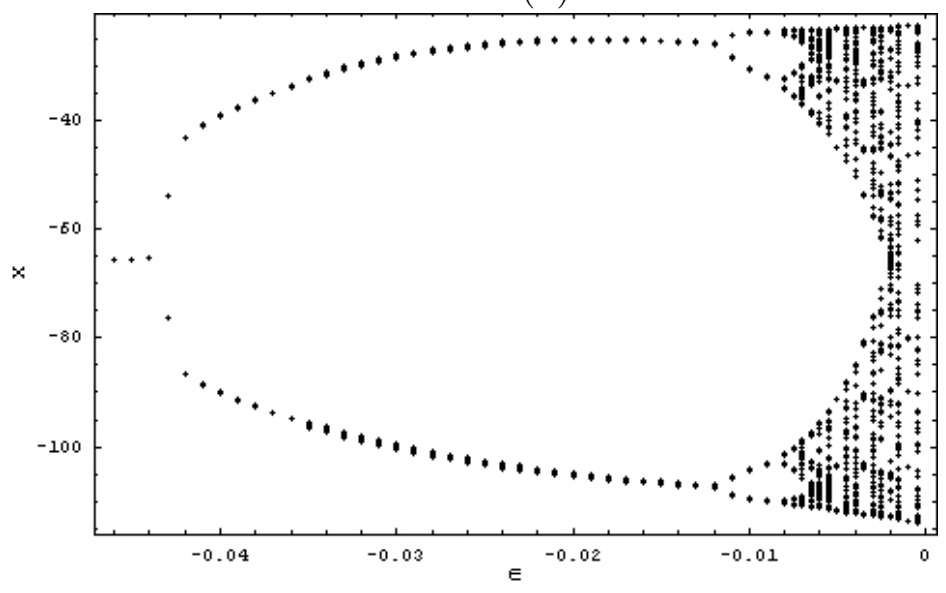

Fig. 4 continuation. 